\title{
FCRAO CO SURVEY OF THE OUTER GALAXY
}

\author{
MARK H. HEYER \\ Five College Radio Astronomy Observatory \\ Lederle Research Building \\ University of Massachusetts at Amherst \\ Amherst, MA 01003
}

\section{Introduction}

FCRAO has initiated an imaging survey of CO $J=1-0$ emission from the outer Galaxy to better understand the physical properties of the molecular gas component of the interstellar medium and to determine the relationship of this component to spiral arm features. When completed in Spring 1996, the CO Survey will cover $103^{\circ}<l_{\mathrm{II}}<145^{\circ},-3^{\circ}<b_{\mathrm{II}}<5^{\circ}$, and $-150<$ $\mathrm{V}_{\mathrm{LSR}}<50 \mathrm{~km} \mathrm{~s}^{-1}$ with angular sampling of $50^{\prime \prime}$ and velocity resolution of $0.8 \mathrm{~km} \mathrm{~s}^{-1}$. During the first year of observations, we have focused upon two fields with longitude ranges of $103^{\circ}<l_{\mathrm{II}}<112^{\circ}$ and $131^{\circ}<l_{\mathrm{II}}<139^{\circ}$. The first field corresponds to the Cepheus region while the second field contains the W3, W4, and W5 complex of HII regions. To date, 700,000 CO spectra have been collected. In this contribution, we present preliminary results from the first year of observations.

\section{Results}

An image of peak antenna temperature $\left(T_{A}^{*}\right)$ of CO $J=1-0$ emission from the Cepheus region over the $\mathrm{V}_{\mathrm{LSR}}$ range of -65 to $+5 \mathrm{~km} \mathrm{~s}^{-1}$ is shown in Figure 1. It includes emission from molecular clouds within the solar neighborhood (distance $<1 \mathrm{kpc}$ ) in addition to clouds associated with the Perseus spiral arm located at a distance of $3.5-5 \mathrm{kpc}$. The bright, high contrast emitting regions within the image are exclusively associated with luminous far infrared sources and optical HII regions. In most cases, the far infrared sources are not the exciting stars of the optical nebulae but rather, newborn stars still deeply embedded within the cloud cores. 


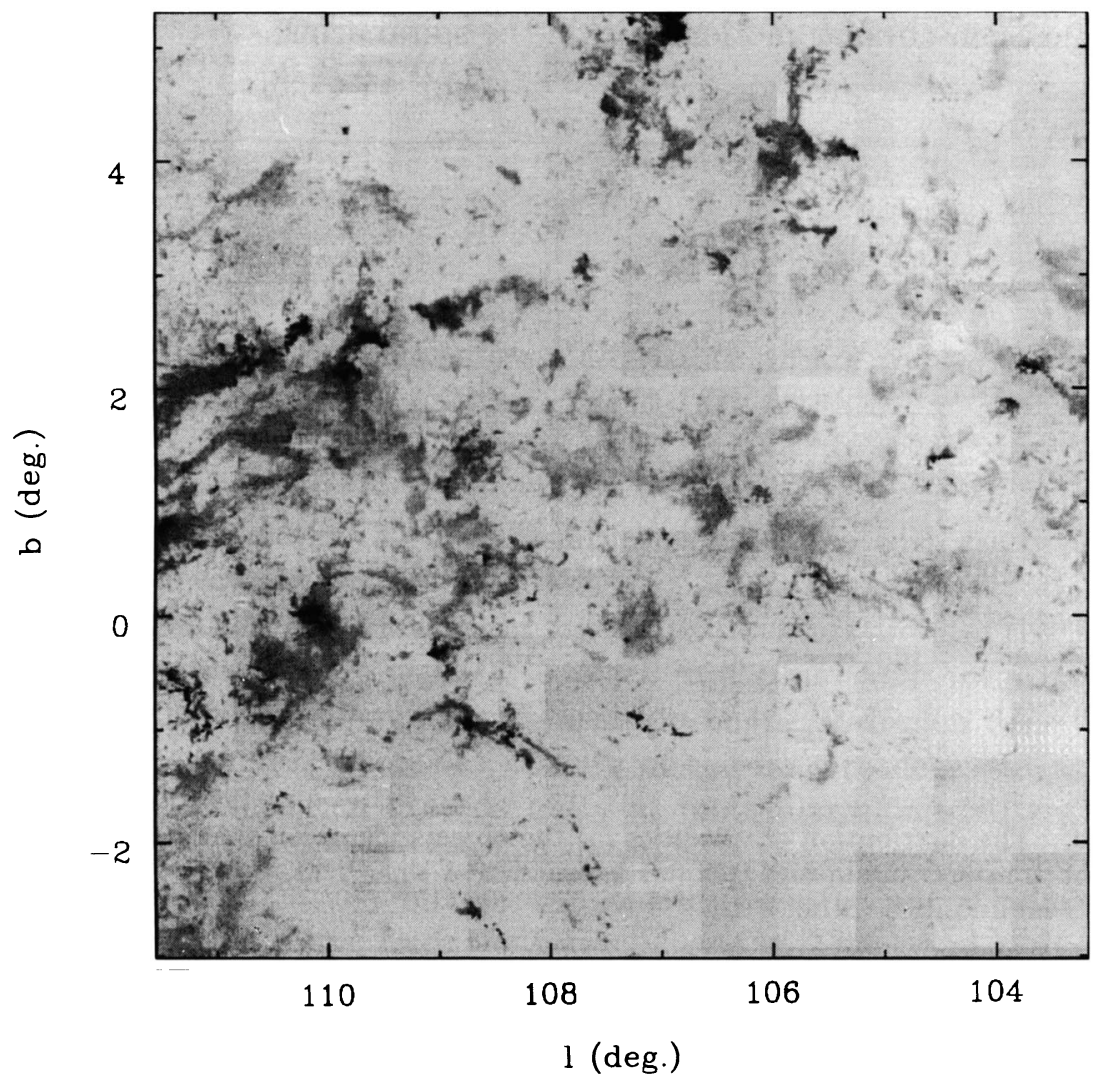

Figure 1. Peak antenna temperature of CO $J=1-0$ emission from the Cep region of the plane of the Galaxy. The image is constructed from 360,000 spectra.

The images provide an unprecedented view into the internal structure and kinematics of the molecular interstellar medium. We have identified 1140 "clouds" which are defined as closed surfaces of antenna temperature within the $l-b-v$ data cube at a threshold of $2 \mathrm{~K}\left(\mathrm{~T}_{R}^{*}\right)$. This is similar to the cloud definitions used by Solomon et al. (1987) and Scoville et al. (1987) but at a much lower threshold of antenna temperature. The distributions of velocities for the identified objects within the two regions are shown in Figure 2. There are strong enhancements in the number of objects at the velocities -10 and $-45 \mathrm{~km} \mathrm{~s}^{-1}$ which correspond to the local spiral arm and the Perseus spiral arm respectively. Within the spiral arms, there is no evidence for large scale coherent velocity structure although radial streaming motions of Perseus spiral arm clouds have been inferred by many 
studies (see Brand and Blitz 1993). We estimate the one dimensional cloudcloud velocity dispersion to be $4-6 \mathrm{~km} \mathrm{~s}^{-1}$. This includes both random and streaming, non-circular motions within the spiral arms.

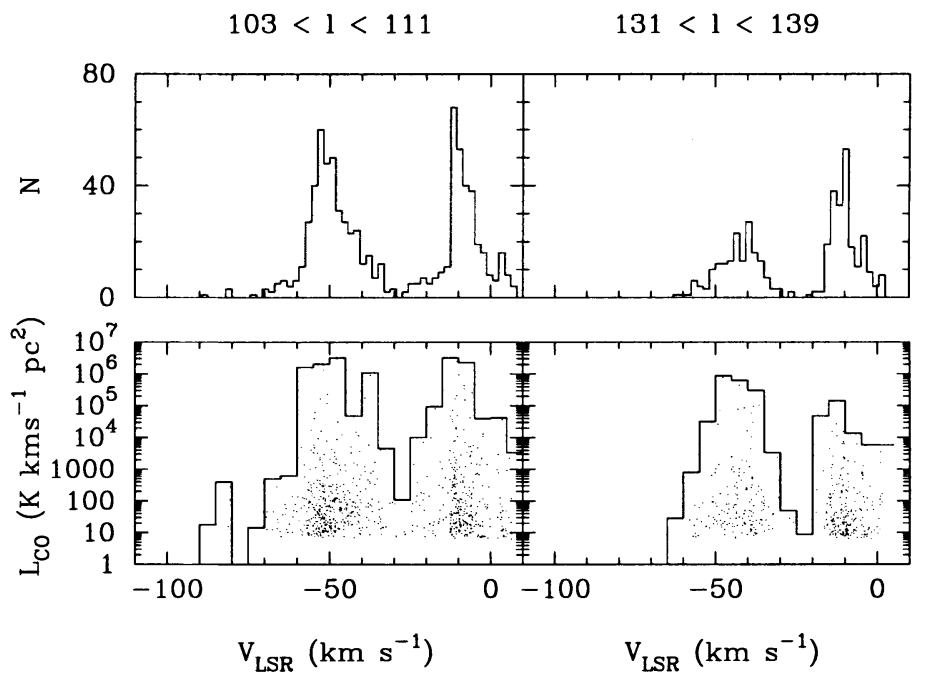

Figure 2. The distribution of velocities for the objects identified within the 2 observed fields. (bottom) CO luminosity (points) as function of $V_{L S R}$. The solid lines are the sum of all CO luminosities within $5 \mathrm{~km} \mathrm{~s}^{-1}$ bin.

Assuming a flat rotation curve in the outer Galaxy, $\Theta_{0}=220 \mathrm{~km} \mathrm{~s}^{-1}$ and $R_{0}=8.5 \mathrm{kpc}$, kinematic distances and CO luminosities are calculated for each identified object. The bottom panels of Figure 2 show the $\mathrm{CO}$ luminosity $L_{\mathrm{CO}}$, for each object as a function of $\mathrm{V}_{\mathrm{LSR}}$ and the sum within each velocity bin. There is a similar strong enhancement of $L_{\mathrm{CO}}$ at the nominal spiral arm velocities. Although we detect clouds at velocities which correspond to interarm regions, the arm-interarm contrast is large in the outer Galaxy. These figures also show that within a given $V_{L S R}$ bin, the largest contribution to the total $\mathrm{CO}$ luminosity is from several large clouds. Only a small fraction of the total $\mathrm{CO}$ luminosity originates from the low level "chaff" of the molecular interstellar medium. Thus, most of the mass of the molecular interstellar medium resides within the small number of very massive clouds.

\section{References}

Brand, J. \& Blitz, L. (1993) The velocity field of the outer Galaxy, $A A$, 275, pp. 67-90. Scoville, N. Z., Yun, M. S., Clemens, D. P., Sanders, D. B., \& Waller, W. H. (1987) Molecular Clouds and Cloud Cores in the Inner Galaxy, ApJ Suppl, 63, pp. 821-915.

Solomon, P. M, Rivolo, A. R., Barret, J., \& Yahil, A. (1987) Mass, Luminosity, and Line Width Relations of Galactic Molecular Clouds, $A p J$, 319, pp. 730-741. 